\title{
Evaluation of the Quality of Aerial Links in Low-Power Wireless Sensor Networks
}

\author{
Jianjun Wen (1D) and Waltenegus Dargie (D), Senior Member, IEEE
}

\begin{abstract}
A wireless sensor network (WSN) assisted by Unmanned Aerial Vehicles (UAVs) can be used to monitor various phenomena in remote, extensive, inaccessible, or dangerous places. The WSN on the ground can provide close-to-thescene sensing, in-network data processing, and multi-hop communication. The UAVs can interface the ground network with a remote control station or facilitate fast and flexible data collection. To this end, the aerial links established between the UAVs and the WSN are critical. The reliability of the links depends on many factors, including Cross Technology Interference (CTI), the relative distance of the UAVs from the ground nodes, the drive quality of the UAVs, and noise. In this paper, we present experimental results addressing some of these issues. Our experiments consisted of eleven IEEE 802.15.4 compliant transceivers, nine of which were deployed on the ground in a grid topology with a further two being attached to a UAV. From careful examination of traces extracted from received packets we concluded that CTI is the most significant factor affecting the quality of aerial links. Our observations have application for deployment related decision-making and for the design of UAV-assisted data collection protocols.
\end{abstract}

Index Terms-experimental measurement, link quality fluctuation, UAV, wireless links, wireless sensor networks

\section{INTRODUCTION}

The combination of Unmanned Aerial Vehicles (UAVs) and Wireless Sensor Networks (WSNs) in remote, extensive, inaccessible, or dangerous places enables a wide range of applications such as monitoring natural disaster [25], detection of potential toxic gas leakages in chemical plants [22], search and rescue operations following man-made as well as natural disasters [5], and precision agriculture [4], [18]. In this setup, each system brings with it a set of advantages and vulnerabilities. The nodes on the ground can provide close-to-the-scene sensing and multi-hop communication, but they may not be able to guarantee a fully-connected ground network. This is particularly the case if the nodes are deployed randomly or in haste. The UAVs, on the other hand, may provide fast, flexible, and safe data collection and command dissemination, but the links they establish with the ground nodes and with each other may be unstable, being affected by factors such as wind and vibration. In addition, both systems operate with exhaustible batteries which limit both duration and quality of operation. The quality of operation and communication of the overall setup may be further affected by cross technology interference (CTI). This is because additional networks (or channels) may be needed to coordinate with the deployment mission and to manage the UAVs.

In the past decade, several researchers have attempted to characterise the quality of low-power wireless links using various metrics, including Received Signal Strength Indicator (RSSI), Link Quality Indicator (LQI), Signal-to-Noise Ratio

Manuscript first submitted on 19 January, 2021.

This work has been partially funded by the Free State of Saxony under TG70 Research Funding program (Grant nummber: 100369691).

Jianjun Wen and Waltenegus Dargie are with the Faculty of Computer Science, Technische Universität Dresden, 01062 Dresden, Germany (email: $\{$ jianjun.wen, waltenegus.dargie\}@tu-dresden.de)
(SNR), and Packet Reception Ratio (PRR) [2], [9], [26]. These studies reveal that even in static scenarios the link quality is predominately bursty, meaning, the links are unstable and packet loss is considerably high. These aspects have significant impact on the end-to-end latency of received packets and the overall lifetime of the network, as a large number of packets have to be retransmitted during communication.

The purpose of this paper is twofold: Firstly, to provide an analysis not only of the quality of ground-to-ground links, but also of aerial-to-ground links. Secondly, all available channels and different interaction modes are considered, thus rendering a more comprehensive analysis. Furthermore, our analysis incorporates the impact of CTI and the metrics which best describe it. Our experiments consisted of nine ground sensor nodes deployed in a grid topology and a commercial drone carrying two on-board sensor nodes, the antennas of which were aligned orthogonally. These nodes interact with the ground nodes in different modes. Our observations are summarised in the following way:

- The radio technology used by the UAV remote controller interfered considerably with the IEEE 802.15.4 compliant transceivers of the wireless sensor nodes. Indeed, $75 \%$ of the available channels suffered from a significant interference. Even though some researchers claim that Channel 26 is the least affected by CTI, we could not confirm this claim. Similarly, the link quality of the IEEE 802.11b channel we set up to coordinate the experiment activities was affected by the UAV's remote controller.

- The quality of ground-to-air link was degraded when the UAV was near to the ground. The link quality in terms of RSSI at first improved as altitude increased, but worsened above a certain threshold. A logarithmic lineof-sight model does not fully account for the path loss in 
this scenario.

- RSSI and PRR decreased as a function of horizontal distance.

- In the presence of CTI, PRR was jointly correlated with RSSI and background noise.

- In a bursty link, more than $99 \%$ of the time the number of packets lost in succession was less than 10 .

- Packet failure was spatially correlated.

- The impact of antenna orientation on PRR was modest.

The remaining part of this paper is organized as follows: In Section [II] we review state-of-the-art. In Section III we describe the experiment setup. In Section IV, we investigate link quality fluctuation across all the sixteen available channels. In Section V, we discuss the spatial characteristics of ground and air links. Then in Section VI we investigate the correlation between PRR, RSSI and noise and their temporal and spatial characteristics. In Section VII we examine the impact of antenna orientation on link quality. In Section IX we provide concluding remarks.

\section{Related Work}

Characterising the quality of wireless links established by low power radios in the ISM band is key in building practical wireless sensor networks and in designing efficient and reliable communication protocols. In the past decade, a considerable effort has been made towards this end [2], [12], [17], [21], [26]. Broadly speaking, we can categorise the state-of-the-art into (1) ground links and (2) aerial links.

\section{A. Ground Links}

Research on the quality of low power wireless links comprises numerous experiments conducted in university laboratory settings [2], [12], [19], [26]. Interestingly, the observations made are often different, making generalisation difficult.

Srinivasan et al. [26] published an in-depth empirical study about low-power wireless links. Their experiments were conducted in multiple testbeds and channels, with different network sizes and deployment environments. According to their observations, (1) interference generated by IEEE $802.11 \mathrm{~b}$ networks caused correlated failure across multiple nodes; (2) link burstyness was correlated with inter-packet-interval; (3) under certain conditions, there was a strong correlation between RSSI and PRR. The authors concluded that observations based on measurements often contradict conceptual models and produce unexpected performance in practical settings.

Mottola et al. [21] presented an extensive empirical study on the performance of low-power wireless links. Their deployment consisted of 20 sensor nodes in two types of tunnels and a vineyard. By analysing more than 320,000 data traces, they found that the links in the tunnels, compared with those in the open field, were more stable, and the communication range, longer. As in [26] and [3], the authors suggested that link asymmetries were observed in most of the wireless links.

Hithnawi et al. [12] investigated the impact of CTI on IEEE 802.15.4 compliant radios using two Tmote Sky nodes in a controlled environment. Thus, the nodes were exposed to different interference sources: a WiFi, a microwave oven, a wireless camera, a Bluetooth transceiver, and a cordless telephone. By examining PRR and the portion of corrupted symbols, they observed that CTI exhibited distinct interference patterns, requiring different strategies to mitigate it. Similar studies [8], [16], [20] were also performed, however, most of them focus on the co-existence of low-power technologies with the IEEE 802.11b technology. Table I] summarises the most important features of the investigations on low-power wireless sensor networks.

\section{B. Aerial Links}

Wireless links connecting a WSN with UAVs, or the nodes they carry, extend the dimensionality of the network, but introduce significant operation and communication dynamics. This is particularly the case if the UAVs are operated by remote controllers.

A few number of UAV deployments have been carried out to investigate the characteristics of the aerial links they establish with wireless sensor networks in outdoor environments. In [13], the authors investigated the stability of ground-to-air links by deploying a pair of TI CC2530 radios, one of which was on the ground while the other was attached to a fixwing UAV. They observed that when the RSSI value was above $-90 \mathrm{dBm}$, a PRR of $80 \%$ could be achieved within the communication range (around 150 meters). Chen et al. [6] observed the same figures over a much shorter communication range (around 30 meters horizontally and 10 to 12 meters vertically).

In [23], the authors employed four ground sensor nodes in a linear topology and a DJI Matrice 100 Quadcopter carrying two IEEE 802.15.4 compliant transceivers to investigate link quality fluctuation. In addition, they investigated the impact of signal obstruction and variations in antenna orientation. They observed that even though the best performance was achieved when the UAV was flying at an altitude of around $45 \mathrm{~m}$. The PRR was above $75 \%$ even when the UAV was flying at a vertical distance of $76 \mathrm{~m}$ and a horizontal distance of $100 \mathrm{~m}$.

In [27], the authors deployed ten TelosB sensor nodes in three farming clusters to monitor various parameters pertaining to precision farming. In order to evaluate the characteristics of the aerial links at different altitudes, a UAV equipped with a TelosB node was employed for collecting packets from the ground network. Their investigation showed that the link quality of the aerial link was better than the ground-to-ground links by $19 \%$ in terms of RSSI and by $70 \%$ in terms of PRR.

Our study complements these investigations by considering different modes of communications and UAV flights, as well as additional statistical metrics. Table $\Pi$ compares Related Work with our work.

\section{METHODOLOGY}

Our experiments were conducted in a large test field next to a forest (as shown in Fig. 1). The ground sensor nodes with IEEE 802.15.4 compatible transceivers were deployed in a grid topology set at interval of 10 meters column-wise and 5 meters row-wise. In addition, two sensor nodes were attached 
TABLE I: Summary of link quality studies in WSNs.

\begin{tabular}{|l|l|l|l|l|l|}
\hline Study & Type & Environment & Network size & Link type & Focus \\
\hline Srinivasan et al. [26] & WSN & office building, campus & 20 to 100 & G-G & characteristics of low power wireless links in stationary WSNs \\
\hline Mottola et al. [21] & WSN & tunnel, vineyard & 20 nodes & G-G & environment impact (tunnels) \\
\hline Hithnawi et al. [12] & WSN & laboratory & 4 & G-G & CTI (WiFi, Bluetooth, wireless camera, cordless phone etc.) \\
\hline Huiru et al. [13] & UAV-WSN & outdoor & 1 ground, 1 air & G-A & link stability of ground to air links \\
\hline Chen et al. [6] & UAV-WSN & outdoor & 1 ground, 1 air & G-A & communication range \\
\hline Nekrasov et al. [23] & UAV-WSN & outdoor & 4 ground, 2 air & G-A & spatial characteristics, antenna orientation etc. \\
\hline Valente et al. [27] & UAV-WSN & outdoor & 10 ground, 1 air & G-A & altitude impact \\
\hline
\end{tabular}

Notes: "G-G": ground to ground link "G-A": ground to air link

TABLE II: Comparison of link quality studies in UAV-WSNs with this work

\begin{tabular}{|c|c|c|c|c|c|c|c|c|c|}
\hline \multirow{2}{*}{ Study } & \multirow{2}{*}{ UAV } & \multirow{2}{*}{ UAV motion } & \multirow{2}{*}{ Network size } & \multirow{2}{*}{ Channel } & \multirow{2}{*}{ CTI } & \multicolumn{2}{|c|}{ Spatial impact } & \multirow{2}{*}{$\begin{array}{l}\text { Failure } \\
\text { correlation }\end{array}$} & \multirow{2}{*}{$\begin{array}{l}\text { Antenna } \\
\text { orientation }\end{array}$} \\
\hline & & & & & & Altitude & distance & & \\
\hline Huiru et al. [13] & 1 fixed wing & hovering & 1 ground, 1 air & 1 & $\mathrm{x}$ & $0-150 \mathrm{~m}$ & $\mathrm{x}$ & $\mathrm{x}$ & $\mathrm{x}$ \\
\hline Chen et al. [6] & 1 quadrotor & - & 1 ground, 1 air & 1 & $\mathrm{x}$ & - & $6-38 \mathrm{~m}$ & $\mathrm{x}$ & $\mathrm{x}$ \\
\hline Nekrasov et al. [23] & 1 quadrotor & straight line trajectory & 4 ground, 2 air & 1 & $\mathrm{x}$ & $9-122 \mathrm{~m}$ & $\max 250 \mathrm{~m}$ & $\mathrm{x}$ & $\checkmark$ \\
\hline Valente et al. [27] & 1 quadrotor & predefined trajectory & 10 ground, 1 air & 1 & $\mathrm{x}$ & - & - & $\mathrm{x}$ & $\mathrm{x}$ \\
\hline This work & 1 quadrotor & hovering, trajectory & 9 ground, 2 air & all 16 & $\checkmark$ & $0-30 \mathrm{~m}$ & $0-100 \mathrm{~m}$ & $\checkmark$ & $\checkmark$ \\
\hline
\end{tabular}

Notes: "-": unknown " $\checkmark$ ": yes "x": no

to a DJI Mavic 2 Enterprise drone The antenna of one of these nodes was oriented vertically, whereas the other was oriented horizontally. We conducted three types of experiment to investigate the quality of the aerial links:

- The UAV hovered at approximately $5 \mathrm{~m}$ height above the ground.

- The UAV varied its vertical distance from a minimum distance of $3 \mathrm{~m}$ to a maximum distance of $30 \mathrm{~m}$.

- The UAV kept its vertical distance at $10 \mathrm{~m}$ and moved horizontally while communication took place.

A detailed description of the experiments will be given in the appropriate sections.

\section{A. Hardware Platforms}

Our wireless sensor network consisted of 11 Zolertia REMote revision $\mathrm{B}$ motes ${ }^{2}$, nine of which were deployed on the ground in a grid topology, whereas two additional nodes were carried by the UAV. The RE-Mote integrated two IEEE 802.15.4 compliant transceivers working on ISM $2.4 \mathrm{GHz}$ and 863-950 MHz. In our experiments we only used the $2.4 \mathrm{GHz}$ band. Each RE-Mote was connected to a Raspberry PI board via a USB cable. The two RE-Motes which were attached to the drone had the same specification as the ground nodes, but they were powered by $3.7 \mathrm{~V}$ LiPo batteries and availed $32 \mathrm{~GB}$ SD cards for storage.

The DJI Mavic 2 Enterprise drone was controlled by a remote controller which used a proprietary long range transmission (OcuSync 2.0 [15]) operating at $2.4 \mathrm{GHz}$ and $5.8 \mathrm{GHz}$ bands. Channel selection inside the remote controller occurred automatically, on the basis of the perceived background noise. As the remote controller shared the $2.4 \mathrm{GHz}$ band with the sensor nodes, the strong transmission signal it generated could cause a significant CTI. In order to achieve a stable and smooth control of the UAV, we set the flight mode on T-mode (tripod) thereby limiting the maximum flight and the ascend/descend speed to $1 \mathrm{~m} / \mathrm{s}$.

\footnotetext{
${ }^{1}$ https://www.dji.com/mavic-2-enterprise

${ }^{2} \mathrm{https}: / /$ zolertia.io/product/re-mote/
}

\section{B. Testbed}

Our experiments were controlled by the MobiLab testbed [28]. MobiLab is a scalable and flexible experiment control framework consisting of a control plane and a WSN testing plane. The control plane is responsible for controlling the execution of an experiment and has a WiFi backbone channel to (1) configure experiment parameters, (2) coordinate interand intra-experiment activities and (3) collect experiment parameters. The DJI Mavic 2 Enterprise drone does not provide any onboard SDK. As a result, the flight motion could not be controlled by MobiLab. To get around this problem, we implemented a waiting time to allow the drone to reach a specific position before communication between the aerial and the ground nodes in each round of the experiment. Communication between the aerial and the ground nodes was coordinated to avoid packet collision. Hence, at any given time, there was only a single transmitter in use.

\section{Link Quality Metrics}

The communication parameters we used to configure the nodes are listed in Table III For all the experiments, the transmission power (tx-power) was set to $7 \mathrm{dBm}$, which is the maximum power level in CC2538 [14]. The inter-packet interval was set to $16 \mathrm{~Hz}$ by default. With this configuration, it took around 3 minutes to transmit 3000 packets. The payload of the packet contained a monotonically increasing counter which was 4 bytes long and the remaining 24 bytes were zeros. We disabled the retransmission feature in the protocol to make sure that each packet was transmitted only once. For most of our experiments, channel 25 was used, since it was the least interfered channel by the DJI remote controller and the $\mathrm{WiFi}$ backbone channel. In the subsequent sections this channel is implicitly referred to unless others stated.

During our experiments, link quality metrics from physical and link layers were recorded. These include RSSI, LQI, ACK, and background noise (before each transmission and after each reception). Besides these, we also estimated PRR and SNR offline. 

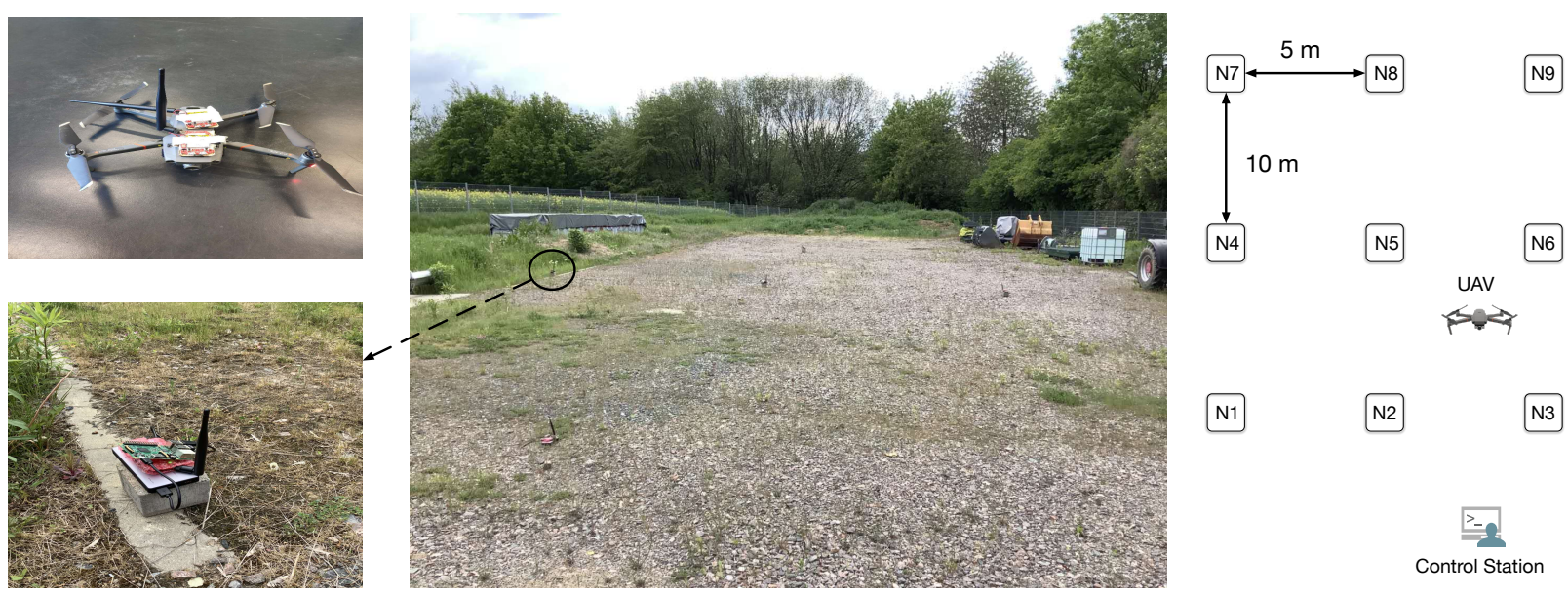

Fig. 1: The deployment setting.

TABLE III: The communication parameter settings for all experiment

\begin{tabular}{|l|l|}
\hline Parameter & Value \\
\hline ipi & 16 or $32 \mathrm{~Hz}$ \\
\hline tx-power & $7 \mathrm{dBm}$ \\
\hline channel & 25 \\
\hline payload length & 28 byte \\
\hline transmissions & 2000 to 3000 \\
\hline
\end{tabular}

\section{Channel EFFEcts}

In the design and evaluation of low-power communication protocols (IEEE 802.15.4) Channel 26 is often set as the preferred channel, due to its supposed robustness against CTI ${ }^{3}$ [12], [26]. Similarly, in the investigation of the performance of aerial links, almost all existing approaches assume that the UAV's control channel is either altogether different [23] or orthogonal to the communication channels used by the wireless sensor nodes [1].

In a previous study [29], we investigated the quality of aerial links employing Channel 26 and observed that this channel suffered from a significant CTI on account of the UAV's remote controller. In this section, we will closely investigate the impact of the UAV's remote controller on all the sixteen channels of the IEEE 802.15.4. In the previous section, we have already highlighted the fact that the DJI remote controller may use an overlapping spectrum. Since the software managing the remote controller (OcuSync 2.0) is proprietary, it is difficult to predict which of the channels the remote controller uses at any given time and which of the IEEE 802.15.4 channels will undergo interference as a result. To investigate this feature, we conducted two sets of experiments: one without the UAV (power off mode) and the other with it.

\section{A. Experiment Setup}

In both setups, the RE-Mote on the UAV with the vertical antenna was configured as a transmitter and the other nodes

\footnotetext{
${ }^{3}$ Channel 26 of IEEE 802.15 .4 is orthogonal to the commonly used WiFi channels 1,6 and 11 .
}

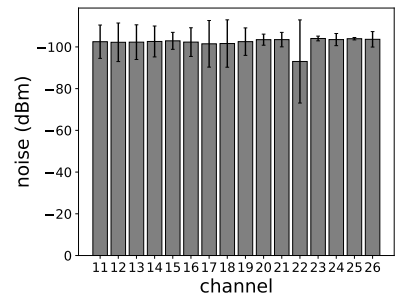

(a)

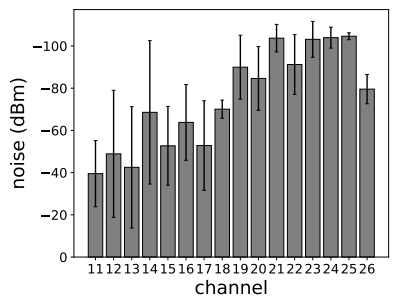

(b)
Fig. 2: Background noise when the UAV was (a) inactive and (b) active.

on the ground as receivers. The transmitter broadcast 2000 packets in burst in each communication channel. We repeated our experiments for all the 16 channels and recorded the link quality metrics. In the second set of experiments the UAV was hovering at around 5 meters height during packet transmission. When the UAV was powered off, the main external interference came from our testbed's WiFi backbone channel.

\section{B. Background Noise}

Fig. 2 shows the average background noise in each channel. The error bars in the plots depict the standard deviation. As can be seen, in the first set of experiments, the background noise in all the channels except in Channel 22 is consistently below $-100 \mathrm{dBm}$ (Fig. 2 (a). Notice that the direction of the $y$-axis is inverted). Compared to the first set of experiments, the background noise increased significantly in most of the channels, as shown in Fig. 2 (b). In addition, unlike the well-regulated WiFi channels, which only affect a handful of IEEE 802.15.4 channels [12], [20] - we observed only a single channel being affected (Channel 22) - the DJI remote controller interfered with $75 \%$ of the channels. Only Channel 21, 23, 24 and 25 were not affected appreciably.

Since CTI was significant on Channel 22 in both experiment sets, we plotted the empirical density function of the background noise in this channel to draw a comparison. Fig. 3 


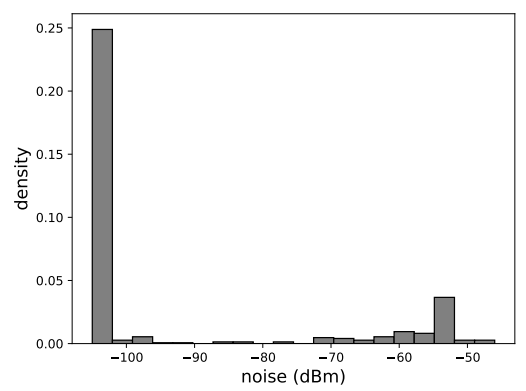

(a)

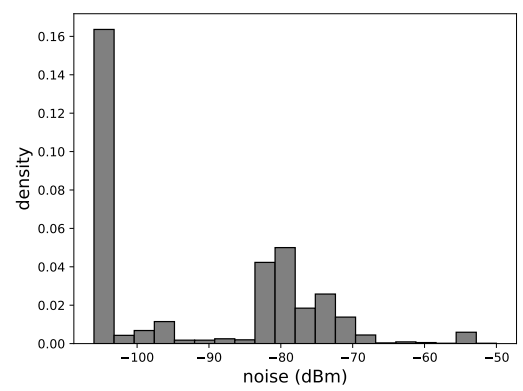

(b)

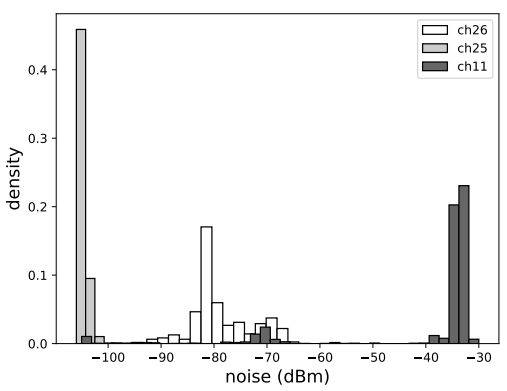

(a)

Fig. 3: Distribution of background noise on Channel 22 when the UAV was (a) inactive and (b) active.

Fig. 4: Distribution of background noise on Channels 11, 25, and 26.

(a) shows the case where the background noise mainly arose from the WiFi backbone channel and Fig. 3 (b) shows the case where both the UAV's controller and the WiFi backbone channel contributed to the interference. Compared to the first case where $74 \%$ of the background noise was below -100 $\mathrm{dBm}$, only $47 \%$ of the background noise was below $-100 \mathrm{dBm}$ when the UAV was active. Moreover, whereas only $23.4 \%$ of the background noise was greater than $-85 \mathrm{dBm}$ in the first case, the portion now increased to $45.9 \%$ in the second case. From this analysis, it can be concluded that when the UAV was active, the dominant external interference came from the radio used to control it. Compared to the IEEE 802.15.4 radios, the DJI remote controller has a much higher transmission power, which reaches up to $20 \mathrm{dBm}$ [10]. Secondly, the WiFi backbone channel was used only occasionally, to deliver control commands, whereas the communication between the UAV and its remote controller was much more frequent.

Amongst all the channels, Channel 11 suffered CTI most (with a mean background noise of $-39.5 \mathrm{dBm}$ and a standard deviation of 15.7). Fig. 4 shows the distribution of background noise on Channel 11, 25, and 26 for comparison. As can be seen, $86 \%$ of the background noise was above $-40 \mathrm{dBm}$ on Channel 11. On Channel 26, 95.7\% of the background noise was between $-90 \mathrm{dBm}$ and $-65 \mathrm{dBm}$.

\section{Received Signal Strength Indicator}

Fig. 5 shows the average RSSI values of the 2000 received packets in each of the sixteen channels, the error bars representing the standard deviation. The plots were generated from the data traces of a receiver which was the nearest to the transmitter on the UAV. The RSSI values in the left figure are on average by around $30 \mathrm{~dB}$ higher compared to those in the right figure. This, however, is due to the difference in the relative distance between the transmitter and the receiver in the two test cases. We will discuss the spatial impact in more details in Section V] Regardless of the difference in absolute values, we observed that, in both cases, the RSSI values were stable, varying only slightly over time (a variation of ca. 7 $\mathrm{dB}$ ). Hence, using RSSI values alone, it is difficult to assert which of the channels attained a better link quality. One of the reasons for this is that the RSSI values of lost packets could not be accounted for since the packets were lost. For example, it is apparent from the preceding subsection that there was a strong CTI on Channel 22 due to both the WiFi backbone links and the DJI remote controller. But the average RSSI values suggest that Channel 22 was slightly better than Channel 25, which, as we have shown, was the channel which suffered CTI the least.

Fig. 6 shows the RSSI values of individual received packets in Channels 26, 25, 22 and 11 for comparison. The RSSI values in the first three channels varied between -40 and $45 \mathrm{dBm}$ in the left plots (when the UAV was inactive) but they varied between -55 to $-65 \mathrm{dBm}$ in the right plots (when the UAV was active), clearly indicating that packets were consistently received with poor quality when the UAV was active.

\section{Packet Reception Ratio}

The PRR is a link quality metric which is widely used to estimate the long-term stability of a communication link. It is computed as the ratio of the number of successfully received packets to the total number of transmitted packets. In this subsection, we will discuss the PRR across all 16 channels, with and without UAV flight. The plots in Fig. 7 show the PRR for the two experiment sets. We observed that when the UAV was inactive, all the packets were successfully received in all the channels with the exception of Channel 22, in which the PRR was around 0.8 (refer to Fig. 7(a)). However, when the UAV was active, the PRR in more than $60 \%$ of the channels was consistently below 0.4 (only in 4 channels was it above 0.98). The channel which is most commonly used in the WSN community, namely, Channel 26, experienced considerable interference, with a PRR of 0.53. Similarly, Channels 11, 12, and 13 exhibited the worst performance, their PRR falling below 0.15 . In other words, more than $85 \%$ of the packets were lost during transmission. Another notable phenomenon is that the PRR decreased in Channel 22, dropping from 0.79 when the UAV was inactive to 0.39 under the compound interference effect of the WiFi backbone links and the UAV's remote controller. 


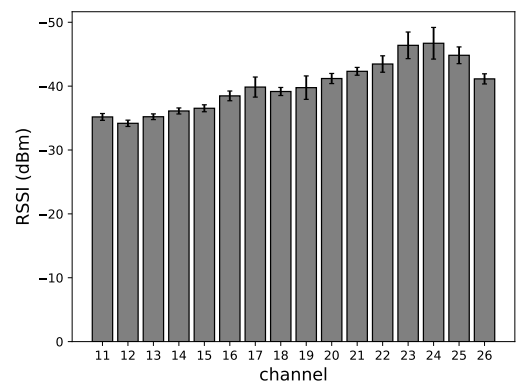

(a)

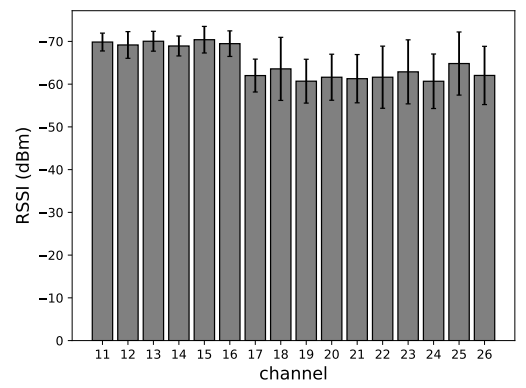

(b)

Fig. 5: The RSSI profile of the 16 channels when the UAV was (a) inactive and (b) active.

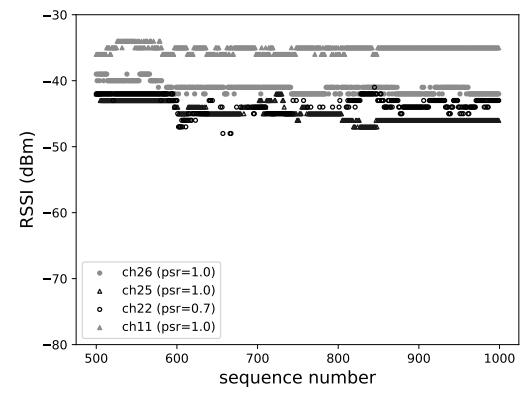

(a)

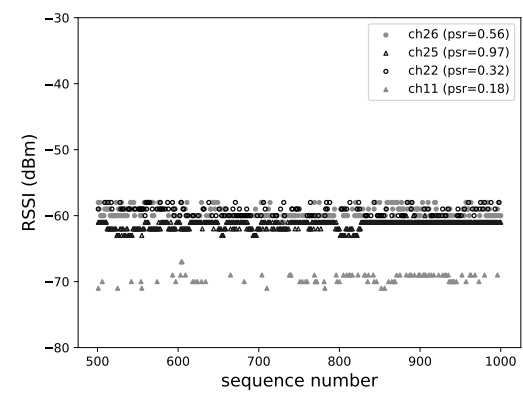

(b)

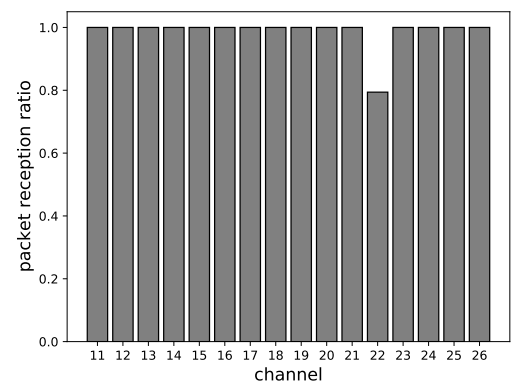

(a)

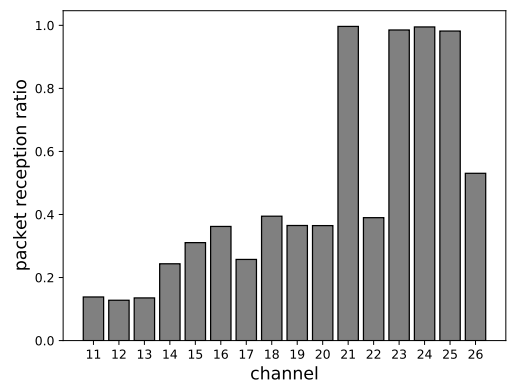

(b)

Fig. 6: RSSI fluctuation in Channel 26, Fig. 7: Packet reception ratio when the 25, 22 and 11 when the UAV was (a) UAV was (a) inactive and (b) active. inactive and (b) active.

\section{SPATIAL CHARACTERISTICS}

The quality and stability of the vertical links a UAV establishes with the ground nodes are directly affected by the UAV's distance from the ground nodes and flight quality. In this section, we will quantitatively evaluate these aspects.

\section{A. Altitude}

We deployed one RE-Mote on the ground and configured it as a transmitter. Another RE-Mote with its antenna vertically oriented was carried by the UAV and set as a receiver. The transmitter transmitted 2000 packets in burst, while the UAV was hovering at specific heights. During transmission, the position of the UAV was fixed, but after transmission, we increased the altitude of the UAV by $1 \mathrm{~m}$ (ranging from 1 to 20 meters; then we considered two additional altitudes, namely, 25 and 30 meters). The horizontal distance between the UAV and the ground node was fixed, which was approximately 3 meters.

Observations: Fig. 8 (a) shows the plots of RSSI values for the different altitudes. The orange marks in the plots denote the median values and the blue plot represents the average value at specific altitudes. As can be seen, the RSSI values at first increased as the altitude increased, reaching a maximum value when the UAV was $8 \mathrm{~m}$ high (refer to Fig. 8 (a)). As the altitude of the UAV increased by $1 \mathrm{~m}$, the RSSI value dropped by around $10 \mathrm{~dB}$. From an altitude of $10 \mathrm{~m}$ to $15 \mathrm{~m}$, the average RSSI decreased slightly. But from a height of $15 \mathrm{~m}$ to $19 \mathrm{~m}$, the average RSSI increased once again. At last, when the UAV reached altitudes of 25 and $30 \mathrm{~m}$, the RSSI decreased as expected. In short, the RSSI variations do not follow the line of sight path loss model, which defines a logarithmic relationship between the RSSI and the transmission distance.

Fig. 8 (b) shows the plot of PRR vs. altitude. We found that when the UAV was flying near the ground (1 to 3 meters), the PRR was very poor, as low as 0.3 . When, on the other hand, the UAV was above $4 \mathrm{~m}$ and up to $19 \mathrm{~m}$, the PRR could exceed $85 \%$. But when the UAV was hovering at $25 \mathrm{~m}$ height or beyond, the PRR fell to a low level once again (less than $0.4)$.

\section{B. Horizontal Distance}

Similarly, we deployed a pair of RE-Motes, one on the ground, transmitting 2000 packets in burst, and another on the UAV, serving as a receiver. In the experiment, the UAV took off next to the ground node and rose to a height of $10 \mathrm{~m}$. Then it flew along a straight path away from the ground node a distance of $100 \mathrm{~m}$. After reaching a distance of $100 \mathrm{~m}$, it hovered until transmission was completed. The transmission of the 2000 packets took around $125 \mathrm{~s}(124.99 \mathrm{~s})$. The estimated time between the transmission of any two successive packets (i.e., IPI) was on average $0.06252 \mathrm{~ms}$, with a variance of 0.00067 . We computed the average RSSI and PRR for every 16 packets (in other words, for a transmission duration of $1 \mathrm{~s}$ ).

Fig. 9 (a) shows the RSSI fluctuations in real time (decreasing as the transmission distance increased). Fig. 9 (b) 


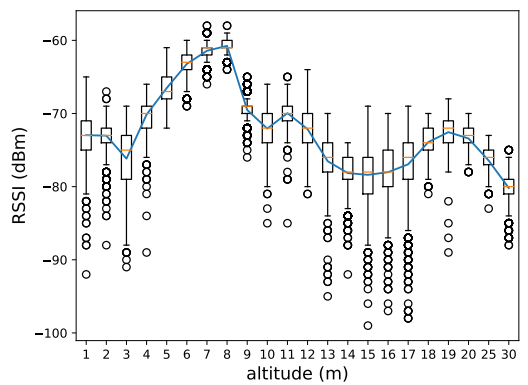

(a)

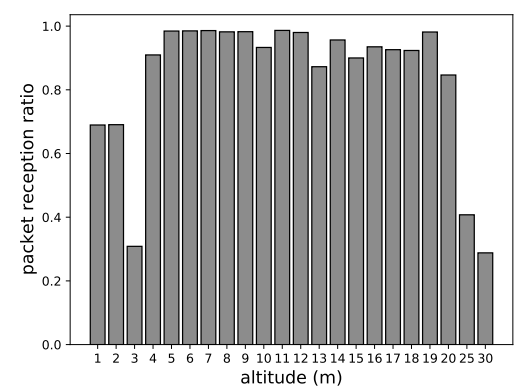

(b)

Fig. 8: Link quality fluctuation as a function of the UAV's altitude.

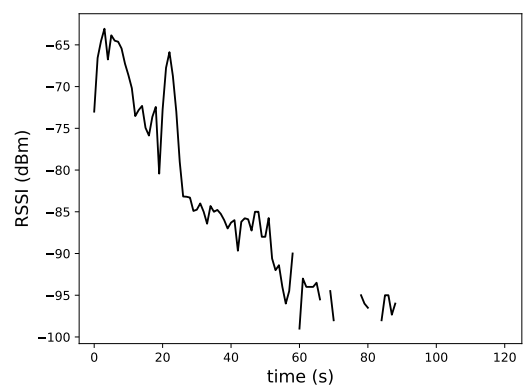

(a)

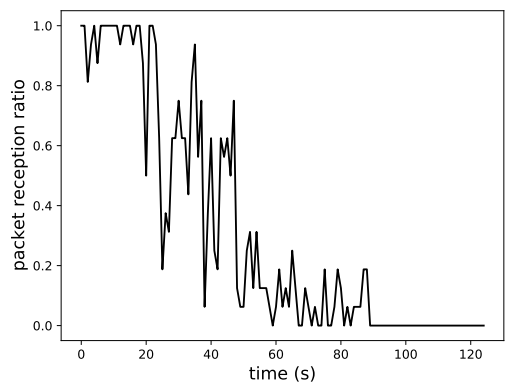

(b)

Fig. 9: Link quality fluctuation as a function of the horizontal distance between a ground node and a UAV.

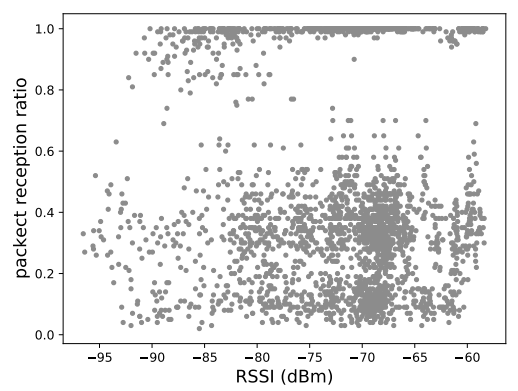

(a)

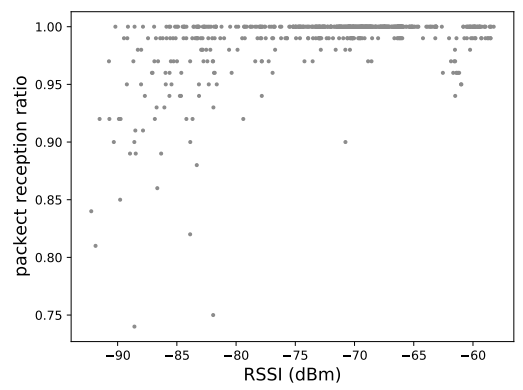

(b)

Fig. 10: The correlation between PRR and RSSI: (a) in all the links. (b) in the links where the noise is less than -97 $\mathrm{dBm}$. shows the PRR variations. As expected, both RSSI and PRR were affected by the relative distance between the transmitter and the receiver. Where the separation distance exceeded $20 \mathrm{~m}$, the PRR became unstable, varying between 0.8 and 0 , dramatically. Where the separation distance exceeded $60 \mathrm{~m}$, the PRR dropped below 0.2. The link was disconnected when the separation distance exceeded $90 \mathrm{~m}$.

\section{FAILURE CORRELATION}

The quality of low power links is often categorised into three regions: good, intermediate (bursty), and disconnected [2], [26]. In the first, the link is considered to be stable and the PRR is above $90 \%$. In the second, the link is supposed to be unstable and the PRR varies widely, between $10 \%$ and $90 \%$. In the third region, the link quality is consistently poor with a PRR below $10 \%$. Researchers have attempted to determine additional thresholds for separating the good region from the intermediate region, even though these thresholds differ from study to study [7], [9], [11], [26]. In this particular study, we were unable to observe a clean threshold above which the PRR was larger than $90 \%$. Nevertheless, we shall examine the correlations between PRR, RSSI, and noise in order to determine whether it is possible to categorise the quality of the aerial links. Moreover, we shall examine whether packet reception failures exhibit temporal and spatial correlations.

\section{A. Relationships between PRR, RSSI, and Background Noise}

The data we employed for the analysis in this section were taken from all the experiments involving an active UAV. The traces were divided into time slots, a slot being the duration needed to transmit 100 packets in succession with an IPI of $16 \mathrm{~Hz}$. For each time slot, we computed the average RSSI values and the PRR, taking into account the packets successfully transmitted in that slot. In other words, the statistics of lost packets were not directly taken into account. This inevitably introduced bias into our analysis, particularly, when a large number of packets were lost in a slot [26].

Observations: Fig. 10 (a) shows the scatter plot relating the average RSSI values with the PRR. It is apparent that the RSSI values exhibit no obvious correlation with the PRR, making it impossible to predict PRR from RSSI. Our observations appear to contradict the assertion that when the RSSI value is approximately $10 \mathrm{~dB}$ larger than the sensitivity of the transceiver, the packet loss rate is less than $10 \%$ [26]. There are two possible reasons for this seemingly contradictory result:

- The bias we mentioned above which is introduced in the calculation of the average RSSI value for each slot.

- The condition under which the assertion is made, namely, low external interference and static deployment.

The second reason is the most probable cause of the discrepancy. To substantiate our assertion, we drew a second scatter plot (Fig. 10 (b)) for those links wherein the background noise 


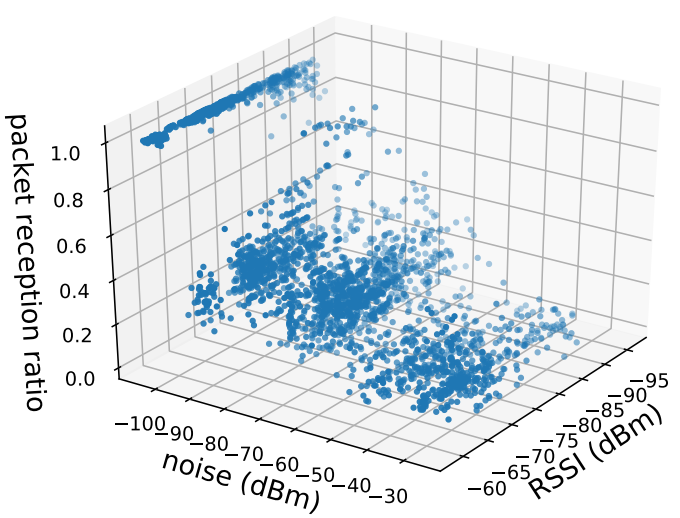

Fig. 11: The correlation between RSSI, noise, and PRR.

was smaller than the radio sensitivity level (i.e., $-97 \mathrm{dBm}$ [14]). It should be remembered that the background noise was sampled before the transmission and after the reception of a packet. From the second plot it is apparent that when the RSSI value is above $-80 \mathrm{dBm}$, the PRR is above $90 \%$, and when the RSSI value is below this threshold, the PRR varies dramatically. To give a more complete view of the correlation of PRR with RSSI and noise, we draw a 3D scatter plot in Fig. 11

Another way to examine the relationship between the three metrics is to compute their multiple correlation coefficient [24]:

$$
R_{p r n}=\sqrt{\frac{r_{p r}^{2}+r_{p n}^{2}-2 r_{p r}^{2} r_{p n}^{2} r_{r n}^{2}}{1-r_{r n}^{2}}}
$$

where $R_{p r n}$ refers to the multiple correlation coefficient; $r_{p r}$, $r_{p n}$ and $r_{r n}$ are the correlation coefficients of PRR and RSSI, PRR and background noise, and RSSI and background noise, respectively. The correlation coefficients are defined as follows 2.

$$
r_{x y}=\frac{\operatorname{cov}(x, y)}{\sigma_{x} \sigma_{y}}
$$

where $\sigma$ is the standard deviation and $\operatorname{cov}(x, y)$ is the covariance of $x$ and $y$. For our case, the correlation coefficient between RSSI and PRR is -0.008 which confirms our conclusion that under a strong CTI, PRR and RSSI are uncorrelated. This said, the multiple correlation coefficient $-R_{p r n}-$ is 0.83 , suggesting that the PRR has a strong association with both RSSI and background noise 4

\section{B. Temporal Correlations}

In the preceding subsection, we discussed the relationship between PRR, RSSI and background noise as an aggregate feature, taking into account statistics from all the links. In this subsection we will explore how the quality of individual links varied over time.

\footnotetext{
${ }^{4}$ The multiple correlation coefficient ranges from 0 to 1,1 representing a perfect linear relationship and 0 the absence of such a relationship
}

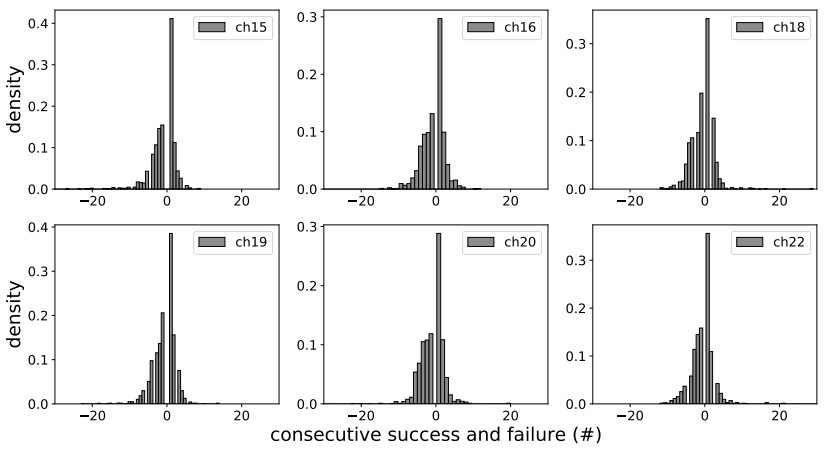

Fig. 12: The distribution of consecutive success and consecutive failure for six channels. Positive values signify consecutive success whereas negative values signify consecutive failure.

For our analysis we rely on the datasets we gathered from Channels 15, 16, 18, 19, 20 and 22 from the experiments described in Section IV (in these channels the PRR is, respectively, $0.31,0.36,0.39,0.37,0.36$ and 0.38). We evaluated the consecutive success and failure rates of packet transmission (CS and $\mathrm{CF}$ ) in each channel. The CS and $\mathrm{CF}$ are a sequence of values generated by counting the number of packets successfully received in succession 5

Observations: CS and CF are metrics useful for evaluating the temporal characteristics of individual links. By analysing their statistical distributions, it is possible to estimate the expected duration of a link in a stable state, in a connected state (in case of CS) or a disconnected state (in the case of CF). The UAV can take advantage of these statistics to determine how long it takes to gather packets from the ground network. Similarly, a dynamic power management strategy can make use of this knowledge to determine the sleep schedule of the nodes on the UAV as well as on the ground.

Fig. 12 shows the distribution functions of CS and CF for the six channels we listed above. In all the plots, a single packet failure and a single packet success are more likely to be experienced in all the channels (the bars near 0 on the $\mathrm{x}$-axis). In more than $99 \%$ of the cases, the number of consecutive failures is less than 10. Likewise, approximately, $99 \%$ of the time, less 10 packets were transmitted in succession (these results may be obtained by integrating the positive and negative regions separately).

\section{Spatial Correlations}

To investigate the existence and nature of spatial correlations in the presence of CTI, we conducted experiments on Channel

${ }^{5}$ Suppose we have a packet reception trace at a receiver, $H=$ $\{0,1,0,1,1,0,0,1,1,1\}$, where 0 signifies a packet reception failure, and 1 represents a packet reception success. Note that in all our experiments, the payload of each packet contains a monotonically increasing Packet Sequence Number, which is used to generate the packet success and failure trace. Then the CS and CF can be computed by counting the number of successes and failures in succession. Thus, we have a series of CS and CF as $S_{\text {cscf }}=$ $\{-1,1,-1,2,-2,3\}$, where the negative signs simply denote the number of packets lost consecutively and the positive signs denote the number of packets received successfully in succession. 


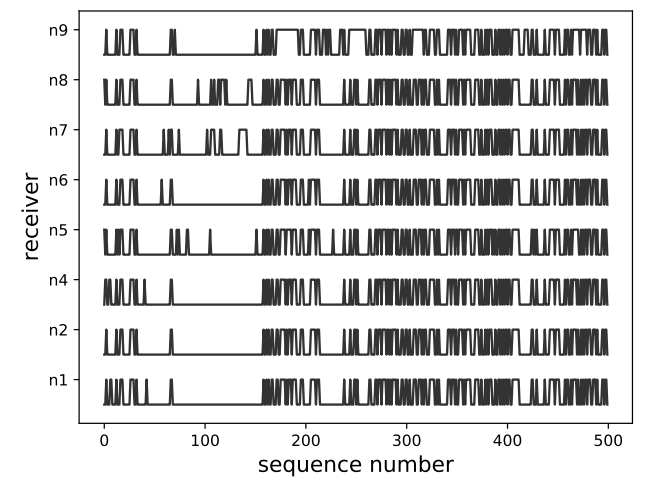

Fig. 13: A snapshot of the packet reception patterns of different receivers. In each plot, the upper band represents success and the lower band represents failure.

26, which suffered moderate CTI. The deployment of the ground sensor nodes and the UAV was as shown in Fig. 1. A single transmitter, carried by the UAV which was hovering at a height of approximately $5 \mathrm{~m}$, broadcast 2000 packets in burst with an IPI of $8 \mathrm{~Hz}$. The ground nodes received the packets and recorded the performance metrics.

Observations: Fig. 13 shows the snapshot of packet receptions patterns for different receivers. It shows that the pattern of packet failure was, by and large, similar for a number of receivers. For example, seven nodes exhibited almost the same packet success and failure patterns between the sequence numbers 400 and 500 . We conclude that the spatial characteristics of aerial links of low-power receivers are most likely correlated. To quantitatively evaluate this assertion, we computed the correlation distance using Equation 3 and plotted the result in Fig. 14 (a).

$$
d(x, y)=1-\frac{\operatorname{cov}(x, y)}{\sigma_{x} \sigma_{y}}
$$

The smaller the correlation distance, the higher is the correlation between any two ground nodes. From the figure, it is apparent that packet reception in all the ground nodes is highly correlated (the minimum correlation distance is 0.24 between nodes 5 and 9), since they all are within the same interference region. Fig. 14 (b) shows the empirical density function of correlated failure. Only $6.4 \%$ of the packet failures occurred exclusively at a single receiver. In other words, more than $93.6 \%$ of the packet failures happened at least at two receivers simultaneously. Up to $43.5 \%$ packet failures were observed at all the ground receivers.

\section{Miscellaneous}

In Section $\mathrm{V}$, we demonstrated that the quality of air-toground links was affected by the height and distance of the UAV with respect to the ground nodes. In this section, we examine whether and to what extent the antenna orientation affects the link quality.

\section{A. Antenna Orientation}

The topology of deployment in this experiment is shown in Fig. 1. Two RE-Motes with different antenna placements were deployed on top of the UAV, the antenna of one of them being vertically oriented whilst the other was horizontally oriented (as shown in Fig. 1, top left). All the nodes on the ground and on the UAV were configured as receivers. Another RE-Mote was placed near the control station (the bottom right in Fig. 11. This node broadcast 3000 packets in burst with an IPI of 8 Hz. The communication channel was Channel 25, which was least affected by CTI. During the experiment, the UAV was hovering at a height of $5 \mathrm{~m}$ above the ground network. The link quality metrics of the ground nodes were gathered using the WiFi backbone channel and transferred to the control station as well as locally stored on a Raspberry PI platform. The data traces of the nodes on the UAV were stored separately on an SD card.

The variation in link quality is explained by the difference in antenna orientation. The plots in Fig. 15 (a) show the temporal variation of the RSSI values of received packets at the nodes deployed on the UAV, while Fig. 15 (b) shows their distribution. Both plots describe two distinct situations, clearly suggesting that the node with the vertical antenna had a consistently better link quality (the difference being more than $15 \mathrm{~dB}$ ). During our experiments, the background noise was around $-104 \mathrm{dBm}$, with a variance of 2.5 . The packet reception ratios were 0.99 at the node with the vertically oriented antenna and 0.92 at the node with the horizontally oriented antenna.

\section{B. Ground-to-Ground versus Ground-to-Air Links}

Fig. 16 compares the quality of ground-to-ground (G-G) and ground-to-air (G-A) links. As expected, the G-A links with the vertical antenna established better links. The average RSSI values of the G-A link with the vertical antenna is more than $10 \mathrm{~dB}$ higher than most of the G-G links, while their PRR was above $99 \%$. This is because the receiver benefited from a line of sight communication much of the time. The ground nodes might also have been affected by the ground reflection. From this figure we can determine that the G-A link for Node 3 was sightly better than all the others. This is because it was the closest node to the transmitter ( $3 \mathrm{~m}$ away).

\section{COMPARISON}

In Section [II] we reviewed state-of-the-art and maintained that our work complements previous work in different ways:

To start with, none of the previous studies seriously addressed CTI introduced by the UAV whereas we paid particular attention to this phenomenon by conducting more comprehensive experiments involving all available channels (16 in all). The observations we made in Section IV reveal that the impact of CTI was appreciable on most of the channels. Even Channel 26 , which is widely used in the literature and supposed to be robust to CTI, was affected.

Secondly, even though RSSI and PRR are widely used metrics to characterise link quality, they, however, were analyzed separately. In this paper, these two metrics are studied 


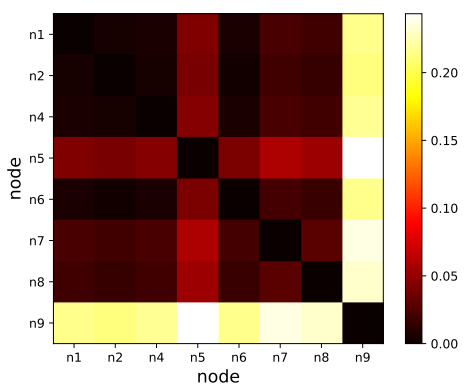

(a)

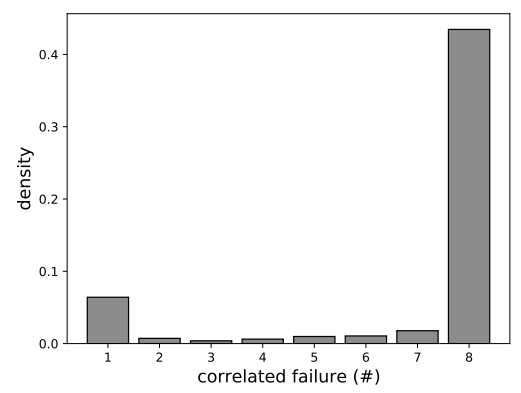

(b)

Fig. 14: The spatial correlations of the packet failure patterns at different receivers using Channel 26: (a) The correlation distance of packet failure. (b) The empirical density function of correlated failure.

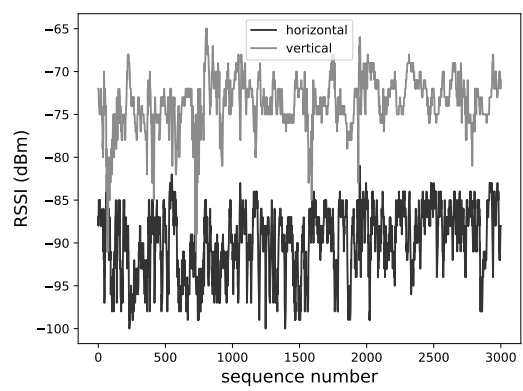

(a)

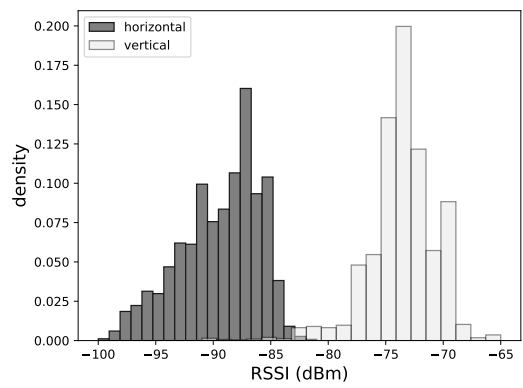

(b)

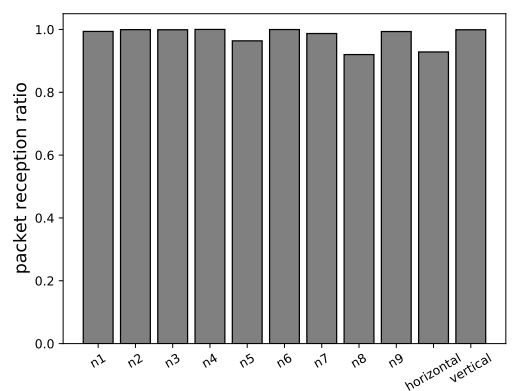

(a)

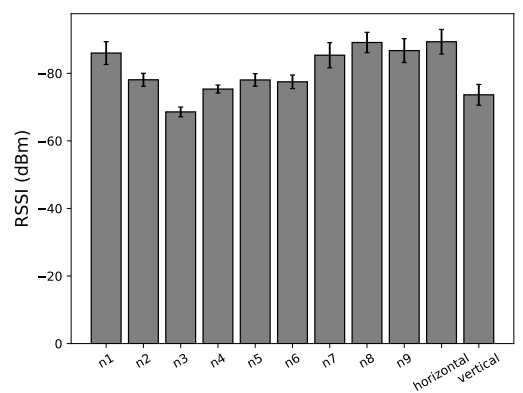

(b)
Fig. 15: The RSSI variations of incom- Fig. 16: Comparison of link quality being packets at the two nodes deployed on tween differently oriented antenna setthe UAV. (a) RSSI fluctuation over time, tings and between ground-to-ground and (b) RSSI distribution. ground-to-air links. (a) PRR, (b) average RSSI. The error bar represents the standard deviation.

both comprehensively (using their statistical distributions) and jointly. The observations we made in Section VI clearly show that PRR is jointly correlated with RSSI and background noise. Additionally, we introduced a new metric (the consecutive success and failure of transmitted packets) to characterise short-term link stability (or burstiness).

Thirdly, except in the case of [23], the impact of antenna orientation on link quality has not been given adequate attention in the analysis of low-power wireless sensor networks. In this work, we not only examined this aspect, but also compared the performance of aerial-to-ground links with ground-toground links. Accordingly, nodes with vertical antenna perform better than nodes with horizontal antenna orientation. Most importantly, our analysis suggests that knowledge of antenna orientation and spatial correlation can be useful for planning the UAV's trajectory and for determining the optimal data collection range.

In terms of observation: Huiru [13] et al. claimed that in their experiment, the aerial links were rather stable, achieving on average a PRR of $80 \%$ even when the RSSI was $-90 \mathrm{dBm}$ and the transmission range, 150 meter. Nekrasov et al. [23] also made a similar claim. We confirm that in the absence of a CTI, these claims are plausible, as we have reported for four of the sixteen channels in Section IV] In the presence of a CTI, however, one has to reckon with a much modest achievement.
Our analysis of the quality of ground-to-ground and groundto-air links in Section VII agrees with the observation made by Valente et al. [27], provided that the node carried by the UAV has a vertically oriented antenna.

\section{CONCLUSiON}

For the purpose of this paper, we conducted various experiments to investigate the characteristics of ground-to-ground and ground-to-air links established by IEEE 802.15.4 compliant transceivers. Similar experiments have been conducted in the past, but they targeted a single channel (Channel 26). Our study examines all the available channels and offers a more comprehensive and practical insight. To the best of our knowledge, ours is the first attempt to study the impact of Cross Technology Interference across the entire spectrum.

Our results reveal that the radio technology used by the UAV has a significant impact on the link quality of IEEE 802.15 .4 compliant radios. Up to $75 \%$ of the channels were subject to a considerable cross technology interference. Additionally, we investigated the impact of the UAV's relative altitude and horizontal distance on the air-to-ground links. The results clearly suggest that to achieve a good performance, for example, a high packet reception ratio, the optimal altitude should be taken into account. This aspect, however, depends on the particular deployment environment. Interestingly, a 
shorter relative distance between a ground node and an aerial node does not necessarily yield a better link quality. Another interesting aspect we observed concerns the temporal and spatial packet failure correlations. Under the same external interference, spatially distributed nodes jointly experienced packet reception failures. A single packet failure was more likely to occur with regard to individual links. More than $99 \%$ of the time, the number of packets which failed in succession was less than 10 . We also studied how link quality was affected by antenna orientation. We observed that an aerial node with a vertical antenna had a better link quality in terms of average RSSI values.

Our study has the following practical applications:

- Understanding patterns of cross technology interference enables implementation of dynamic channel assignment and channel selection.

- Implementation of an efficient and dynamic multichannel communication protocol can mitigate CTI.

- Knowledge of the temporal and spatial patterns of packet failures can enable the identification of an efficient UAV trajectory.

In this study, we have not investigated the impact of the UAV's flight quality on the quality of the aerial links. Furthermore, the quality of air-to-air links remains to be investigated, if multiple UAVs are to be employed to gather data from ground nodes. These will be the focus of our future work.

\section{RefERENCES}

[1] N. Ahmed, S. S. Kanhere, and S. Jha. On the importance of link characterization for aerial wireless sensor networks. IEEE Communications Magazine, 54(5):52-57, 2016.

[2] N. Baccour, A. Koubâa, L. Mottola, M. A. Zúñiga, H. Youssef, C. A. Boano, and M. Alves. Radio link quality estimation in wireless sensor networks. ACM Transactions on Sensor Networks, 8(4):1-33, sep 2012.

[3] N. Baccour, A. Koubâa, H. Youssef, and M. Alves. Reliable link quality estimation in low-power wireless networks and its impact on tree-routing. Ad Hoc Networks, 27:1-25, 2015.

[4] A. D. Boursianis, M. S. Papadopoulou, P. Diamantoulakis, A. LiopaTsakalidi, P. Barouchas, G. Salahas, G. Karagiannidis, S. Wan, and S. K. Goudos. Internet of things (iot) and agricultural unmanned aerial vehicles (uavs) in smart farming: A comprehensive review. Internet of Things, page 100187, 2020.

[5] D. W. Casbeer, R. W. Beard, T. W. McLain, S.-M. Li, and R. K. Mehra. Forest fire monitoring with multiple small uavs. In Proceedings of the 2005, American Control Conference, 2005., pages 3530-3535. IEEE, 2005.

[6] C.-J. Chen, J.-A. Chen, and Y.-M. Huang. Intelligent environmental sensing with an unmanned aerial system in a wireless sensor network. International Journal on Smart Sensing \& Intelligent Systems, 10(3), 2017.

[7] W. Dargie and J. Wen. A seamless handover for wsn using lms filter. In $L C N$, pages 442-445. IEEE, 2014.

[8] I. S. A. Dhanapala, R. Marfievici, S. Palipana, P. Agrawal, and D. Pesch. Modeling wifi traffic for white space prediction in wireless sensor networks. In 2017 IEEE 42nd Conference on Local Computer Networks (LCN), pages 551-554. IEEE, 2017.
[9] X. Ding, G. Sun, G. Yang, and X. Shang. Link investigation of ieee 802.15. 4 wireless sensor networks in forests. Sensors, 16(7):987, 2016.

[10] DJI. Mavic 2 enterprise series user manual, 2020.

[11] H. Fotouhi, M. Zúñiga, M. Alves, A. Koubâa, and P. Marrón. Smarthop: A reliable handoff mechanism for mobile wireless sensor networks. In EWSN, pages 131-146. Springer, 2012.

[12] A. Hithnawi, H. Shafagh, and S. Duquennoy. Understanding the impact of cross technology interference on ieee 802.15. 4. In Proceedings of the 9th ACM international workshop on Wireless network testbeds, experimental evaluation and characterization, pages 49-56, 2014.

[13] C. Huiru, L. Xuanjiao, L. Yeqian, L. Guo, et al. Design and experiment of a wsn sink platform based on fixed wing unmanned aerial vehicles. In 2016 IEEE Advanced Information Management, Communicates, Electronic and Automation Control Conference (IMCEC), pages 12711274. IEEE, 2016.

[14] T. I. Incorporated. Cc2538 powerful wireless microcontroller systemon-chip for 2.4-ghz ieee 802.15.4, 6lowpan, and zigbee applications, 2015.

[15] B. Kyle. Dji ocusync vs. dji lightbridge - what's the difference?, 2018.

[16] P. Li, Y. Yan, P. Yang, X.-Y. Li, and Q. Lin. Coexist wifi for zigbee networks with fine-grained frequency approach. IEEE Access, 7:135363-135376, 2019.

[17] C.-J. M. Liang, N. B. Priyantha, J. Liu, and A. Terzis. Surviving wifi interference in low power zigbee networks. In Proceedings of the 8th ACM Conference on Embedded Networked Sensor Systems, pages 309-322. ACM, 2010.

[18] P. K. R. Maddikunta, S. Hakak, M. Alazab, S. Bhattacharya, T. R. Gadekallu, W. Z. Khan, and Q.-V. Pham. Unmanned aerial vehicles in smart agriculture: Applications, requirements and challenges. arXiv preprint arXiv:2007.12874, 2020.

[19] E. Miluzzo, X. Zheng, K. Fodor, and A. T. Campbell. Radio characterization of 802.15. 4 and its impact on the design of mobile sensor networks. In European Conference on Wireless Sensor Networks, pages 171-188. Springer, 2008.

[20] M. Mohammad, X. Guo, and M. C. Chan. Oppcast: Exploiting spatial and channel diversity for robust data collection in urban environments. In Proceedings of the 15th International Conference on Information Processing in Sensor Networks, page 19. IEEE Press, 2016.

[21] L. Mottola, G. P. Picco, M. Ceriotti, Ş. Gună, and A. L. Murphy. Not all wireless sensor networks are created equal: A comparative study on tunnels. ACM Transactions on Sensor Networks (TOSN), 7(2):1-33, 2010.

[22] M. Mukherjee, L. Shu, L. Hu, G. P. Hancke, and C. Zhu. Sleep scheduling in industrial wireless sensor networks for toxic gas monitoring. IEEE Wireless Communications, 24(4):106-112, 2017.

[23] M. Nekrasov, R. Allen, I. Artamonova, and E. Belding. Optimizing 802.15. 4 outdoor iot sensor networks for aerial data collection. Sensors, 19(16):3479, 2019.

[24] J. Neter, M. H. Kutner, C. J. Nachtsheim, and W. Wasserman. Applied linear statistical models, volume 4. Irwin Chicago, 1996.

[25] M. T. Rashid, D. Y. Zhang, and D. Wang. Socialdrone: An integrated social media and drone sensing system for reliable disaster response. In IEEE INFOCOM 2020-IEEE Conference on Computer Communications, pages 218-227. IEEE, 2020.

[26] K. Srinivasan, P. Dutta, A. Tavakoli, and P. Levis. An empirical study of low-power wireless. ACM Transactions on Sensor Networks (TOSN), $6(2): 16,2010$

[27] J. Valente, D. Sanz, A. Barrientos, J. d. Cerro, Á. Ribeiro, and C. Rossi. An air-ground wireless sensor network for crop monitoring. Sensors, 11(6):6088-6108, 2011.

[28] J. Wen, Z. Ansar, and W. Dargie. Mobilab: A testbed for evaluating mobility management protocols in wsn. In Testbeds and Research Infrastructures for the Development of Networks and Communities, pages 49-58. Springer, 2016.

[29] J. Wen and W. Dargie. Characterization of the link quality of a coordinated wireless environment. In IoT'20 Companion. ACM, 2020 